\title{
Erratum to: Evaluating quantitative and conceptual models of speech production: how does SLAM fare?
}

\author{
Grant M. Walker ${ }^{1}$. Gregory Hickok ${ }^{1}$
}

Published online: 2 February 2016

(C) Psychonomic Society, Inc. 2016

\section{Erratum to: Psychon Bull Rev}

DOI: 10.3758/s13423-015-0962-9

In our article (Walker \& Hickok, 2015), we incorrectly reported that the amount of the Semantic-Lexical-Auditory-Motor (SLAM) model's fit improvement over the SemanticPhonological (SP) model for conduction aphasia was significantly correlated with the conduction patients' lexical bias. Recall that our definition of lexical bias is $\ln (\mathrm{F}+\mathrm{M}+.5 / \mathrm{N}-$ $\mathrm{AN}+.5$ ), where $\mathrm{N}$ is the inclusive category of all nonwords. In our calculation of the lexical bias odds ratio, we erroneously used the counts of Abstruse Neologisms, nonwords that do not share any phonemes with the target, rather than the intended Nonwords, which do. The correct result for the correlation between SLAM fit improvement and lexical bias in conduction aphasia is non-significant $(r=-.10, p=.50)$. The result is still non-significant when Mixed errors are excluded ( $r=.17$, $p=.23$ ). Instead, we find that the strongest predictor of SLAM fit improvement over SP is the combined rate of Formal and Nonword errors $(r=.70, p<1 \mathrm{e}-7)$. These results leave open the possibility, raised by Goldrick (2015), that SLAM's im-

The online version of the original article can be found at http://dx.doi.org/ 10.3758/s13423-015-0962-9.

Gregory Hickok gshickok@uci.edu

1 Cognitive Sciences, University of California, San Francisco, CA, USA provement over SP may be a consequence of SLAM capturing post-lexical phonological errors.

Although we cannot rule out the Lexical + Post-Lexical (LPL) explanation for the conduction naming pattern, our other arguments that disambiguate the SLAM account from LPL remain valid. To test our assumption that SLAM assigns a higher probability of lexical Formal errors in conduction aphasia over SP, we compared the average probabilities of lexical Formal errors between the models with a paired, 1-tailed, ttest. The results were significant $(p=.03 ; d f=47)$, indicating that SLAM assigns a higher probability of lexical Formal errors in conduction aphasia. Combined with weak connections to motor output, this also increases the probability of phonologically-related Nonword errors, offering an alternative explanation than LPL for this naming pattern. Our simulations demonstrated that the phonological feedback was the critical mechanism for inducing the effect, while simply adding another processing level does not. Therefore, despite the lack of overt lexical bias in the naming data, the SLAM model still provides a mechanistic explanation for the observed naming patterns in conduction aphasia that is clearly distinguishable from LPL.

\section{References}

Goldrick, M. (2015). Integrating SLAM with existing evidence: Comment on Walker and Hickok (2015). Psychonomic Bulletin \& Review, 1-5. doi: 10.3758/s13423-015-0946-9

Walker, G. M., \& Hickok, G. (2015). Evaluating quantitative and conceptual models of speech production: how does SLAM fare? Psychonomic Bulletin \& Review, 1-8. doi: 10.3758/s13423-0150962-9 